\title{
Quantitative Electroencephalogaphy (QEEG) and Neurofeedback Training (NFT) for Elderly with Mild Cognitive Impairment (MCI)
}

\author{
Nur Hartini Mardan ${ }^{1 *}$, Norsiah Fauzan ${ }^{2}$ \\ ${ }^{1}$ P.G Student, Faculty of Cognitive Sciences and Human Development, Universiti Malaysia Sarawak \\ ${ }^{2}$ Assc. Prof, Faculty of Cognitive Sciences and Human Development, Universiti Malaysia Sarawak \\ *Corresponding author E-mail:hartinimardan@gmail.com
}

\begin{abstract}
Neurofeedback training (NFT) has been widely used to alter the brain activity to enhance the brain function. This study aimed to apply neurofeedback to enhance the cognitive performance in elderly with Mild Cognitive Impairment (MCI) by focusing on alpha wave in the neurofeedback training as it is positively associated with cognitive decline in elderly. 10 subjects who passed the criteria were assigned to experimental and control group. With 15 sessions of alpha neurofeedback, increase in alpha absolute power was rewarded while simultaneous suppression of theta and beta2 were done in experimental group. Results showed that after completion of neurofeedback, all subjects in experimental group learn to increase their alpha absolute power while mixed result was recorded for suppression of theta and high beta either at individual, inter and intra group level. Cognitive results in individual level revealed that pattern of increase and decrease of score was regular in experimental group and at grouped level, significant increment observed in Digit Span and Symbol Search in experimental group only. These results suggest that MCI elderly could learn to increase specific components of EEG activity that such enhanced activity may facilitate in working memory and processing speed enhancement.
\end{abstract}

Keywords: Cognitive enhancement; elderly; mild cognitive impairment; neurofeedback; QEEG

\section{Introduction}

Cognitive performance of elderly will decline with age and this make them more susceptible to get age related cognitive impairment disease such as mild cognitive impairment and even worse it may lead to dementia $[1,2]$. With the yearly increasing number in $\mathrm{AD}$ cases worldwide, MCI has became a serious issue to be investigated further to find ways to at least slowing its progression to AD. Non-pharmacological rehabilitation technology such as neurofeedback training has caught researchers' attention to curb this problem. Neurofeedback training (NFT) is a self regulation technique that helps individual learns to control or change their brain activity [3] which has been proved to improve elderly cognitive performance $[4,5,6]$.

A highlight on alpha and theta rhythms are given because individuals with greater cognitive impairment present a greater amount of theta activity than is normal in aging individuals $[7,8]$ significant reduction alpha frequency is responsible for slowing down processing speed and memory in particular [9]. Alpha may be trained alone or it is combined with other rhythms such as theta and beta. In an exploratory study, peak alpha frequency (PAF) and alpha amplitude were inhibited and enhanced in two different arrangements in 35-38 sessions; (i) PAF inhibited, alpha amplitude rewarded (ii) PAF rewarded, alpha amplitude inhibited with one mock feedback control. In (i), it was shown that the elderly subjects had improvements in memory while in (ii) there was a general improvement in speed of processing and executive function [4]. Meanwhile another study of alpha training [10] depicted it was possible to increase alpha power even in short period in elderly despite of their aging condition. The study was carried out with 30 participants of elderly individuals (65-85 year-old) in which they were trained in 4 sessions ( 30 minutes per session) in a week to increase in upper-alpha $(10-12 \mathrm{~Hz})$ power while inhibiting theta $(4-7 \mathrm{~Hz})$. Significant increase in alpha power was seen in 8/10 and increase in alpha/theta ratio while there was a non-significant decrease in theta seen.

Theta protocol neurofeedback in literature often revolved around cognitive impairment problem which is the reason it is often done in elderly. Beccera et al. (2012) aimed to reduce the theta absolute power on individualized selected electrode and observed neurofeedback effectiveness in terms of changes in theta absolute power in EEG and improvement in WAIS -revised and NEUROPSI test [5]. The study disclosed the improvement in verbal comprehension index \& verbal IQ of WAIS - revised and NEUROPSI test after 30 training sessions of neurofeedback in experimental group than in control group. Reduction in theta absolute power in the midline and left frontal leads could have induced the improvement in attention that was reflected in the total score of the NEUROPSI test. Despite of widely applied neurofeedback training in research setting, the training for elderly are less explored and it is even less for elderly with particular condition such as as mild cognitive impairment. Thus, in the present study, alpha training (training up alpha and training down theta $\&$ high beta) will be employed to see the effect of training in cognitive performance in elderly with mild cognitive impairment in terms of working memory, processing speed concurently with its EEG changes before and after the training. 


\section{Method}

\subsection{Subject}

Examination M-MMSE-S [11,12] score 20 - 26 (iii) obtained PHQ-9 and GAD-7 score less than 10 (iii) self complain of memory problems (iv) able to perform pre and post task, WAISIV (v) completed elementary school. Only 10 people managed to participate until the end of the study, with only 5 people in experimental and the same 5 people on control group.

\subsection{Cognitive Instrument}

For the pre and post task assessment of cognitive performance, digit span \& arithmetic from Working Memory Index (WMI) and symbol search \& coding subtest from Processing Speed Index (PSI) of Weschler-IV, were used.

\subsection{Qeeg Recording and Analysis}

The machine used in this study was Mitsar EEG with 21 channels QEEG cap was positioned on scalp according to 10-20 International System with earlobes as references. After obtaining the electrical activity from the scalp it was amplified then digitized The computation of spectral analysis was performed by Fast Fourier Transform algorithms. This study used WinEEG software developed by Mitsar. The details of analysis followed the guide from Quantitative EEG, Event-Related Potentials and Neurotherapy [13].

\subsection{Neurofeedback Training}

The NFT machine used in this study was known as Brain Train from Spectrum Learning. Active electrodes were placed according to $10-20$ system at T3, T4, C3, C4, P3 and P4 and the ground and reference electrodes were attached at left and right earlobe respectively. Neurofeedback training (NFT) was carried out for 15 sessions per subject in experimental group. Each training session lasted for 30 minutes. They were trained to increase alpha power while suppressing the theta and high beta.

\subsection{Statistical Analysis}

Statistical analysis involved inter-subject analysis for experimental group and control group separately. This was done using Wilcoxon Signed Rank Test given that the number of subjects in this research was relatively small to use parametric test. Intra-subject analysis with Mann Whitney test was done to compare the differences in experimental and control group in terms of changes in pre and post QEEG reading and cognitive results.

\section{Result}

\subsection{Sociodemographic Data}

Table 1: Sociodemographic Data

\begin{tabular}{|l|l|l|}
\hline \multicolumn{2}{|c|}{ Table 1: Sociodemographic Data } \\
\hline Variable & Experimental & Control \\
\hline Age & $60.2 \pm 2.4$ & $63.3 \pm 3.5$ \\
\hline Gender & $\mathrm{M}=3, \mathrm{~F}=2$ & $\mathrm{M}=3, \mathrm{~F}=2$ \\
\hline Education years & $8.6 \pm 2.5$ & $10 \pm 7.04$ \\
\hline MMSE score* & $23.20 \pm 0.84$ & $25.40 \pm 0.894$ \\
\hline PHQ-9 & $3.40 \pm 4.5$ & $3.00 \pm 3.6$ \\
\hline GAD-7 & $0.80 \pm 1.3$ & $2.40 \pm 3.6$ \\
\hline
\end{tabular}

This final analysis involved 10 elderly subjects who voluntarily agreed to continue on participating with mean age of 60.2 years old $(\mathrm{SD}=2.4)$ for experimental group and 63.3 years old $(\mathrm{SD}=$ $3.5)$. There were no significant differences in education years (Exp: $\mathrm{M}=8.6$ years, $\mathrm{SD}=2.5$; Cont: $\mathrm{M}=10, \mathrm{SD}=7.04$ ), Patient Health Questionnaire - 9 (PHQ-9) (Exp: $\mathrm{M}=3.4, \mathrm{SD}=4.5$; Cont: $\mathrm{M}=3.0, \mathrm{SD}=3.6)$ and Generalized Anxiety Disorder- 7 (GAD-7) questionnaire (Exp: $\mathrm{M}=.80, \mathrm{SD}=1.3$; Cont: $\mathrm{M}=2.4, \mathrm{SD}=3.6$ ). Mini Mental State Examination (MMSE) score shown a statistical difference between experimental and control group $(\mathrm{Z}=-2.471, \mathrm{p}$ $=.016$ ).

\subsection{QEEG Changes}

\subsubsection{Intra-Group}

In eyes closed condition, experimental participants show significant increment in median by $0.287 \mathrm{uV}^{2}$ at the end of the training $(z$ $=-2.023, p=.043)$ at $\mathrm{C} 3 \mathrm{C} 4$ for theta even though theta was suppressed during the training. Training up alpha in T3T4, C3C4 and P3P4 locations caused escalation of alpha absolute power by 0.339 $\mathrm{uV}^{2}, 5.055 \mathrm{uV}^{2}$ and $1.88 \mathrm{uV}^{2}$ respectively $(z=-2.023, p=.043)$ while training down high beta was only successful in one location, at T3T4 with decrease in median by $1.790 \mathrm{uV}^{2}(z=-2.023, p$ $=.043$ ). Control participants shows increment in theta at $\mathrm{P} 3 \mathrm{P} 4$ by $0.585 \mathrm{uV}^{2}$ in post QEEG and decrease in alpha at P3P4 by 2.15 $\mathrm{uV}^{2}(z=-2.023, p=.043)$. Note that the $\mathrm{p}$-value for the significant results are the same; 0.043 due to the same value of mean rank/sum of ranks.

\subsubsection{Inter-Group}

Significant results (Table 2) were observed in alpha absolute power at $\mathrm{C} 3 \mathrm{C} 4$ for experimental group was significantly higher than for control group, $(\mathrm{U}=2.00, \mathrm{p}=.028$. $)$ This was also observed at P3P4 with experimental group absolute power was greater control $(\mathrm{U}=0.00, \mathrm{p}=.009)$. Beta2 absolute power at T3T4 for experimental and control group were notably different, $(\mathrm{U}=2.00, \mathrm{p}$ $=.028)$.

\subsection{Cognitive Changes}

\subsubsection{Inter-Group}

In experimental group, NFT elicited an increase in the post Digit Span score in all 5 subjects. The test determined that there was a statistically significant median increase in digit span score (1.00), $\mathrm{z}=-2.121, \mathrm{p}=.034$. Control group showed lower pre score (23.00) than post score $(19.00)$ decreased $(-2.00)$ in digit span score in 4 out of 5 but no significant difference was observed, $\mathrm{z}=-1.518, \mathrm{p}$ $=.129$. A Wilcoxon signed-rank test determined there was no significant median increase (3.00) in experimental group in Arithmetic score, $\mathrm{z}=-1.633, \mathrm{p}=.102$. Control group showed unchanged pre and post score (9.00) with 2 of 5 had decreased in score, another 2 had the same score while 1 showed increase in score with no significant difference was observed, $\mathrm{z}=-0.816, \mathrm{p}$ $=.414$. In Symbol Search test, a significant median increase by 3.00 was observed in experimental group (pre $=22.00$, post $=$ 25.00 ) in symbol search score, $\mathrm{z}=-2.023, \mathrm{p}=.043$. Control group showed no distinguish difference $\mathrm{z}=-1.483, \mathrm{p}=.138$. There were no significant median increase (6.00) in experimental group $\mathrm{z}=$ $1.219, \mathrm{p}=.223$ and in control group, no change in median observed, $\mathrm{z}=-0.730, \mathrm{p}=.465$ for Coding.

\subsubsection{Intra-Group}

No significant differences in all cognitive tests as reported in Table 3 .

\begin{tabular}{|c|c|c|c|c|c|c|c|}
\hline & & & $\begin{array}{c}\text { Rank } \\
\text { Average }\end{array}$ & $\begin{array}{c}\text { Sum of } \\
\text { Ranks }\end{array}$ & $\mathbf{U}$ & $\mathbf{Z}$ & p \\
\hline \multirow{6}{*}{$\alpha$} & \multirow{2}{*}{ T3T4 } & $\mathbf{E}$ & 6.00 & 30.00 & \multirow{2}{*}{10.00} & \multirow{2}{*}{-0.522} & \multirow{2}{*}{.602} \\
\hline & & $\mathrm{C}$ & 5.00 & 25.00 & & & \\
\hline & \multirow{2}{*}{ C3C4 } & $\mathbf{E}$ & 7.60 & 38.00 & \multirow{2}{*}{2.00} & \multirow{2}{*}{-2.193} & \multirow{2}{*}{$.028^{*}$} \\
\hline & & C & 3.40 & 17.00 & & & \\
\hline & \multirow{2}{*}{ P3P4 } & $\mathbf{E}$ & 8.00 & 40.00 & \multirow{2}{*}{0.00} & \multirow{2}{*}{-2.661} & \multirow{2}{*}{$.009^{*}$} \\
\hline & & $\mathrm{C}$ & 3.00 & 15.00 & & & \\
\hline$\theta$ & T3T4 & $\mathbf{E}$ & 5.60 & 28.00 & 12.00 & -0.104 & .917 \\
\hline
\end{tabular}




\begin{tabular}{|c|c|c|c|c|c|c|c|}
\hline & & $\mathbf{C}$ & 5.40 & 27.00 & & & \\
\hline & $\mathrm{ClC}_{4}$ & $\mathbf{E}$ & 6.80 & 34.00 & 600 & -1358 & 175 \\
\hline & $\cos 4$ & C & 4.20 & 21.00 & 0.00 & -1.030 & .17/ \\
\hline & & $\mathbf{E}$ & 5.20 & 26.00 & & & \\
\hline & P3P4 & $\mathbf{C}$ & 5.80 & 29.00 & 11.00 & -0.315 & .154 \\
\hline & & $\mathbf{E}$ & 3.40 & 17.00 & & & \\
\hline & 1314 & C & 7.60 & 38.00 & 2.00 & -2.193 & .028 \\
\hline & & $\mathbf{E}$ & 5.20 & 26.00 & 1100 & -0313 & 754 \\
\hline B & C $3 \mathrm{C} 4$ & $\mathbf{C}$ & 5.80 & 29.00 & 11.00 & -0.313 & .154 \\
\hline & P3P4 & $\mathbf{E}$ & 5.60 & 28.00 & 1200 & -0104 & 917 \\
\hline & 1514 & $\mathbf{C}$ & 5.40 & 27.00 & & & (911 \\
\hline
\end{tabular}

result after neurofeedback training is evidence that the adjustment of brain function is not as easy with neurofeedback [19] neither in elderly nor in young people.

The suppression of beta2 yielded mix result at individual and grouped level. Beta frequency range was stated to be from $13 \mathrm{~Hz}$ to $30 \mathrm{~Hz}$, with beta1 at $13-16 \mathrm{~Hz}$, beta 2 at $16-20 \mathrm{~Hz}$ and beta3 at $20-28 \mathrm{~Hz}$ [20]. From the analysis of frequency of all subjects' average range was $19-20 \mathrm{~Hz}$ and it fallen into beta2 range. Suppression of beta 2 were often paired with other brain rhythm components such as SMR $(12-15 \mathrm{~Hz})$ and theta. It was seldom be a

Table 3: Cognitive result

\begin{tabular}{|c|c|c|c|c|c|c|}
\hline & & $\begin{array}{c}\text { Rank } \\
\text { Average }\end{array}$ & $\begin{array}{l}\text { Sum of } \\
\text { Ranks }\end{array}$ & $\mathbf{U}$ & $\mathbf{Z}$ & p \\
\hline \multirow{2}{*}{ Digit span } & $\operatorname{Exp}$ & 7.10 & 35.50 & \multirow{2}{*}{4.50} & & \multirow{2}{*}{.082} \\
\hline & Cont & 3.90 & 19.50 & & 1.741 & \\
\hline \multirow{2}{*}{ Arithmetic } & Exp & 7.20 & 36.00 & \multirow{2}{*}{4.00} & & \multirow{2}{*}{.074} \\
\hline & Cont & 3.80 & 19.00 & & 1.786 & \\
\hline \multirow{2}{*}{$\begin{array}{l}\text { Symbol } \\
\text { search }\end{array}$} & Exp & 4.90 & 24.50 & \multirow{2}{*}{9.50} & - & \multirow{2}{*}{.530} \\
\hline & Cont & 6.10 & 30.50 & & 0.629 & \\
\hline \multirow{2}{*}{ Coding } & Exp & 5.60 & 28.00 & \multirow{2}{*}{12.00} & - & \multirow{2}{*}{.917} \\
\hline & Cont & 5.40 & 27.00 & & 0.105 & \\
\hline
\end{tabular}
main component to be discussed extensively in most neurofeedback studies contributing to its lack of report regarding its effect when it was suppressed. However, the combinations of beta2 with others simultaneously during neurofeedback showed beta2 suppression was successful [21] as had been seen in the current study.

\subsection{Alpha and Cognitive Performance}

The present study aimed to observe the changes in cognitive performance in terms of working memory (Digit span, Arithmetic) and processing speed (Symbol search, Coding) by means of conducting Weschler-IV (WAIS-IV) test before and after commenc-

\section{Discussion}

\subsection{Effect of NFT to Absolute Power}

The absolute power measure in QEEG showed alpha activity increased whether in individual or grouped result in experimental group but not in control group which did not received any kind of intervention. In intra-group result, significant difference was observed in all locations; T3T4, C3C4 and P3P4 in experimental group. In inter-group result, there was significant difference in between experimental and control group observed in central $(\mathrm{C} 3 \mathrm{C} 4)$ and parietal areas (P3P4) but not in T3T4. Among all three locations (T3T4, C43C4 and $\mathrm{P} 3 \mathrm{P} 4)$ either in inter or intragroup $\mathrm{P} 3 \mathrm{P} 4$ has the highest alpha absolute power changes because it is known that posterior area has the strongest alpha rhythms and known to be dominated by well-known alpha sources in parietaloccipital sulcus [14]. Beside, alpha activity was produced by two generators; one posterior (parieto-occipital) and one more central (centro-parietal), with reciprocal connections and even mutual competition for neuronal resources [15]. The selections of central posterior sites were based on this nature of fact. Hence by taking advantage of this condition at central posterior areas, it was easier to increase alpha rhythms. This explained the results particularly in inter-group result and also the reason P3P4 had the highest absolute power changes in intra-group result. The present finding adds to the growing body of literature on the alpha trainability when alpha neurofeeback was applied even though the time course of training could be considered as short as only 15 sessions were done $[16,17]$. The change in alpha activity was thought to be permanent as reported by van Boxtel et al., (2012) who did the follow up after 3 months of NFT. This might explained the effect of alpha NFT to cognitive performance in the experimental group in the next section.

The suppression of theta yielded mix result at individual with majority of the subjects had increment in theta absolute power. It could be concluded in this study the suppression of theta was unsuccessful. In cognitive enhancement training, theta was usually inhibited [7] because its excessive theta activity was related to cognitive impairment. Contrarily to this study, most study yielded successful result when theta was inhibited not only in terms of absolute power or relative power but also in behavioral or cognitive changes $[5,18]$. The failure to suppress theta in this study corroborated with earlier alpha/theta training which failed to suppress theta activity in 4 out of 10 elderly in neurofeedback group [10]. This result was not only observed in the elderly group but in a group of healthy young individuals who failed to decrease theta in an attempt to increase alpha \& suppress theta [6]. The mixed QEEG. In attempt to improve cognitive performance in various domains, alpha rhythms has always been using as an indicator because alpha oscillation has been positively correlated with mental performance at all ages, both in healthy individuals and in individuals with neurological conditions [22].

Alpha neurofeedback whether as unselective alpha band (8-12 $\mathrm{Hz}$ ) or selective alpha band training either upper, lower alpha, individual alpha had shown improvement in cognitive performance in various domains such as working memory, attention and etc. This study chose to apply unselective alpha band training because $9-12 \mathrm{~Hz}$ alpha activity was linked to the working memory task [14] and in memory scanning task [9] even though most study used upper alpha to enhance the cognitive performance . Besides that, elderly with probable MCI or MCI, had shown to have decrement in both alpha lower and upper alpha; with more decrement in lower alpha as observed in Sloreta [23]. The average of alpha frequency range in this study was $8.7 \mathrm{~Hz}-$ $10 \mathrm{~Hz}$ which show overlapping between lower and upper alpha frequency, thus, separating lower and upper alpha power was not a choice.

This study employed general or unselective alpha band training and resulted in promising outcome in experimental group as compared to control group. Intra-grouped result showed significant increase in Digit span and Symbol search in experimental group while inter-group result displayed nearly significant differences in between experimental and control group. To author's knowledge, there are only two studies applying neurofeedback in unselective alpha $(8-12 \mathrm{~Hz})$ to increase cognitive performance. Nan et al., 2012 carried out neurofeedback on adult aged 20-29 years old with individual alpha band $(8-12 \mathrm{~Hz})$ at $\mathrm{Cz}$ location for a total of 20 sessions [24]. The results of this study coincide with Nan et al. where after the neurofeedback, there was an improvement in forward and backward of digit span score in NFT group and they were significantly larger than those of control group. Even though this study and the mentioned study had different target groups; elderly and young adult respectively, elderly and young adult could enhance their cognitive state after neurofeedback training $[10,25]$ even though the rate of learning might be different due to nature of cognitive differences. Angelakis et al. rewarded increase in alpha amplitude in one of its training conditions. As the result, increment in alpha amplitude $(8-12 \mathrm{~Hz})$ was reported to enhance memory in 3-back task performance (working memory measure) and visual memory [4]. The increase in working memory measures might depict the increase in processing speed because 
both were causatively linked to cognitive decline in old age and processing speed was known to moderating the relationship between age and WM [26,27,28].

The main limitation of this study is the small number of participants who are committed to participate until the end. The number of neurofeedback sessions in this study was considered as short (15 sessions) as compared to other studies [4,5,24].

\section{Conclusion}

The effect of neurofeedback might be more robust in terms of absolute power changes and cognitive results if the numbers of sessions are increased. In terms of protocol, if separate protocol for each alpha, theta and beta 2 is carried out for different groups, the changes in absolute power and its relationship with cognitive results will be clearer even though it is well known of alpha relation to cognitive performance in general. It could be concluded that only alpha uptraining was successful. In conclusion, the changes recorded in QEEG particularly in alpha absolute power and in cognitive results were due to neurofeedback.

\section{Acknowledgement}

This work used qeeg machine sponsored by the Ministry of Higher Education Malaysia under grant nos. FRGS/SS02(01)1072/2013(18)

\section{References}

[1] Baker, M., Akrofi, K., Schiffer, R., \& Boyle, M. W. (2008). EEG patterns in mild cognitive impairment (MCI) patients. The Open Neuroimaging Journal, 2(1), 52-55.

[2] Rosdinom, R., Fazli, A., Ruzyanei, N. J., Azlin, B., \& Srijit, D. (2011). Factors associated with cognitive impairment in patients with Parkinson disease: an urban study. Clinical Therapeutics, 162(1), 23-29.

[3] Frank, D. L., Khorshid, L., Kiffer, J. F., Moravec, C. S., \& McKee, M. G. (2010). Biofeedback in medicine: who, when, why and how? Mental Health in Family Medicine, 7(2), 85-91.

[4] Angelakis, E., Stathopoulou, S., Frymiare, J. L., Green, D. L., Lubar, J. F., \& Kounios, J. (2007). EEG neurofeedback: a brief overview and an example of peak alpha frequency training for cognitive enhancement in the elderly. The Clinical Neuropsychologist, 21(1), 110-129.

[5] Becerra, J., Fernández, T., Roca-Stappung, M., Díaz-Comas, L., Galán, L., Bosch, J., ... Harmony, T. (2012). Neurofeedback in healthy elderly human subjects with electroencephalographic risk for cognitive disorder. Journal of Alzheimer's Disease, 28(2), $357-$ 367.

[6] Hanslmayr, S., Sauseng, P., Doppelmayr, M., Schabus, M., \& Klimesch, W. (2005). Increasing individual upper alpha power by neurofeedback improves cognitive performance in human subjects. Applied Psychophysiology and Biofeedback, 30(1), 1-10.

[7] Penttilä, M., Partanen, J. V., Soininen, H., \& Riekkinen, P. J. (1985). Quantitative analysis of occipital EEG in different stages of Alzheimer's disease. Electroencephalography and Clinical Neurophysiology, 60(1), 1-6.

[8] Prichep, L. S., John, E. R., Ferris, S. H., Reisberg, B., Almas, M. Alper, K., \& Cancro, R. (1994). Quantitative EEG correlates of cognitive deterioration in the elderly. Neurobiology of Aging, 15(1), 85-90.

[9] Klimesch, W. (1999). EEG alpha and theta oscillations reflect cognitive and memory performance: a review and analysis. Brain Research. Brain Research Reviews, 29(2-3), 169-195

[10] Lecomte, G., \& Juhel, J. (2011). The Effects of Neurofeedback Training on Memory Performance in Elderly Subjects. Psychology, 2(8), 846-846.

[11] Ibrahim, N. M., Shohaimi, S., Chong, H.-T., Rahman, A. H. A. Razali, R., Esther, E., \& Basri, H. B. (2009). Validation Study of the Mini-Mental State Examination in a Malay-Speaking Elderly Population in Malaysia. Dementia and Geriatric Cognitive Disorders, 27(3), 247-253. https://doi.org/10.1159/000203888

[12] Razali, R., Baharudin, A., Jaafar, N. R. N., Sidi, H., Rosli, A. H. Hooi, K. B., ... Elias, N. A. (2012). Factors associated with mild cognitive impairment among elderly patients attending medical clinics in Universiti Kebangsaan Malaysia Medical Centre. Sains Malaysiana, 41(5), 641-647.

[13] Kropotov, J. D. (2010). Quantitative EEG, Event-Related Potentials and Neurotherapy. Academic Press.

[14] Jensen, O., Gelfand, J., Kounios, J., \& Lisman, J. E. (2002). Oscillations in the Alpha Band (9-12 Hz) Increase with Memory Load during Retention in a Short-term Memory Task. Cerebral Cortex, 12(8), 877-882. https://doi.org/10.1093/cercor/12.8.877

[15] Thatcher, R.W., Krause, P.J., Hrybyk, M., (1976) Cortico-cortical associations and EEG coherence: a two-compartment model. Electroencephalography and Clincical Neurophysiology 64, 123-143

[16] Cho, M. K., Jang, H. S., Jeong, S.-H., Jang, I.-S., Choi, B.-J., \& Lee, M.-G. T. (2008). Alpha neurofeedback improves the maintaining ability of alpha activity. Neuroreport, 19(3), 315-317. https://doi.org/10.1097/WNR.0b013e3282f4f022

[17] van Boxtel, G. J. M., Denissen, A. J. M., Jäger, M., Vernon, D., Dekker, M. K. J., Mihajlović, V., \& Sitskoorn, M. M. (2012). A novel self-guided approach to alpha activity training. International Journal of Psychophysiology: Official Journal of the International Organization of Psychophysiology, 83(3), 282-294. https://doi.org/10.1016/j.ijpsycho.2011.11.004

[18] Vernon, D. J. (2005). Can neurofeedback training enhance performance? An evaluation of the evidence with implications for future research. Applied Psychophysiology and Biofeedback, 30(4), 347 364. https://doi.org/10.1007/s10484-005-8421-4

[19] Sterman, M. B. (2000). Basic concepts and clinical findings in the treatment of seizure disorders with EEG operant conditioning. Clinical EEG (Electroencephalography), 31(1), 45-55.

[20] Rangaswamy, M., Porjesz, B., Chorlian, D. B., Wang, K., Jones, K. A., Kuperman, S., ... Begleiter, H. (2004). Resting EEG in offspring of male alcoholics: beta frequencies. International Journal of Psychophysiology, 51(3), 239-251. https://doi.org/10.1016/j.ijpsycho.2003.09.003

[21] Vernon, D., Egner, T., Cooper, N., Compton, T., Neilands, C., Sheri, A., \& Gruzelier, J. (2003). The effect of training distinct neurofeedback protocols on aspects of cognitive performance. International Journal of Psychophysiology, 47(1), 75-85. https://doi.org/10.1016/S0167-8760(02)00091-0

[22] Klimesch, W. (1997). EEG-alpha rhythms and memory processes. International Journal of Psychophysiology ,26, 319-340

[23] Babiloni, C., Visser, P. J., Frisoni, G., De Deyn, P. P., Bresciani, L., Jelic, V., ... Nobili, F. (2010). Cortical sources of resting EEG rhythms in mild cognitive impairment and subjective memory complaint. Neurobiology of Aging, 31(10), 1787-1798. https://doi.org/10.1016/j.neurobiolaging.2008.09.020

[24] Nan, W., Rodrigues, J. P., Ma, J., Qu, X., Wan, F., Mak, P.-I., ... Rosa, A. (2012). Individual alpha neurofeedback training effect on short term memory. International Journal of Psychophysiology: Official Journal of the International Organization of Psychophysiology, 86(1), 83-87. https://doi.org/10.1016/j.ijpsycho.2012.07.182

[25] Wang, J.-R., \& Hsieh, S. (2013). Neurofeedback training improves attention and working memory performance. Clinical Neurophysiology: Official Journal of the International Federation of Clinical Neurophysiology, 124(12), https://doi.org/10.1016/j.clinph.2013.05.020

[26] Fry, A. F., \& Hale, S. (1996). Processing Speed, Working Memory, and Fluid Intelligence: Evidence for a Developmental Cascade. Psychological Science, 7(4), 237-241. https://doi.org/10.1111/j.1467-9280.1996.tb00366.x

[27] Kail, R., \& Salthouse, T. A. (1994). Processing speed as a mental capacity. Acta Psychologica, 86(2-3), 199-225.

[28] Nettelbeck, T., \& Burns, N. R. (2010). Processing speed, working memory and reasoning ability from childhood to old age. Personality and Individual Differences, 48(4), 379-384. https://doi.org/10.1016/j.paid.2009.10.032 